\title{
Comparison of culture-negative and culture-positive sepsis or septic shock: outcomes are more influenced by the nature of the infectious agent itself than by the samples' positivity
}

Romain Jouffroy ${ }^{1}\left[1\right.$ and Benoit Vivien ${ }^{2^{*}}$

To the editor,

Recently in the Journal, Li et al. [1] reported that culture positivity or negativity was not associated with differences in mortality, intensive care unit length of stay (LOS), mechanical ventilation requirements and renal replacement requirements of sepsis or septic shock patients. Conversely, hospital length of stay and mechanical ventilation duration of culture-positive septic patients were longer than those of culture-negative patients. While the authors must be congratulated for this systematic review and meta-analysis, we believe that their interpretation of the results requires caution. Firstly, we are surprised that for a major issue of interest such as blood culture positivity during sepsis, only seven studies were included by the authors in the final analysis. For example, the study by Vincent et al. [2], which is a 1-day, prospective, multicenter point reporting the prevalence of ICU infection, has not been included here, where $70 \%$ of their patients had positive microbial isolates. Secondly, despite international guidelines [3] on sepsis management, we

This comment refers to the article available online at https://doi.org/10.1186/ s13054-021-03592-8.

\footnotetext{
${ }^{*}$ Correspondence: benoit.vivien@aphp.fr

${ }^{2}$ SAMU de Paris, Service d'Anesthésie Réanimation, Hôpital Universitaire

Necker - Enfants Malades, Assistance Publique - Hôpitaux de Paris, and Paris University, Paris, France

Full list of author information is available at the end of the article
}

cannot exclude heterogeneity between strategies and cares directly influencing outcomes. Thirdly, two studies $[4,5]$ include approximatively 19,000 patients, i.e., $85 \%$ of all cases, which could noticeably influence the results of this meta-analysis. Last but not least, we believe that more than the positivity or negativity of blood sampling, it is more the infectious agent itself that influences the outcomes, especially since the broad spectrum of the initial antibiotic therapy may be responsible for culture negativity.

Nevertheless, beyond all these limitations, we fully agree with $\mathrm{Li}$ et al. [1] that larger-scale studies are required to confirm or infirm their results.

\section{Response to: Comparison of culture-negative and culture-positive sepsis or septic shock: outcomes are more influenced by the nature of the infectious agent itself than by the samples' positivity \\ Yuting $\mathrm{Li}^{3}$, Jianxing Guo ${ }^{3}$, Hongmei Yang ${ }^{3}$, Hongxiang $\mathrm{Li}^{3}$, Yangyang Shen ${ }^{3}$ and Dong Zhang ${ }^{3 *}$ \\ *Correspondence: zhangdong@jlu.edu.cn \\ ${ }^{3}$ Department of Intensive Care Unit, The First Hospital of Jilin University, Changchun 130021, Jilin, China}

We would like to thank Dr. Jourffroy and Dr. Vivien for their interest in our manuscript [1] and for their comments in response to it. Mortality and other clinical 
outcomes between culture-negative and culture-positive septic patients have been documented inconsistently and are very controversial. That's why we performed a systematic review and meta-analysis to compare the clinical outcomes of culture-negative and culture-positive sepsis or septic shock.

Dr. Jourffroy et al. argued that the study by Vincent et al. [2] should be also included in the final analysis. However, as we all know that the most important principle of topic selection for meta-analysis is to have comparison. In this meta-analysis, we also clearly explained our inclusion and exclusion criteria as follows: (1) prospective and retrospective cohort studies; (2) patients with sepsis or septic shock; (3) all authors reported our primary outcome of all-cause mortality; (4) clearly comparing culture-negative versus culture-positive patients with clinically relevant secondary outcomes. We excluded studies including not estimable data and without clear comparisons of the outcomes. In addition, we excluded letter and review. The study by Vincent et al. is a 1-day, prospective, multicenter point reporting the prevalence of ICU infection. In other words, it is a cross-sectional study but not a cohort study, so it didn't clearly compare culture-negative versus culture-positive patients with clinical outcomes. This study met our exclusion criteria but did not meet the inclusion criteria, that's why we didn't include it in the meta-analysis. We admit that the number of included cohort studies is small and we hope that there will be more studies in this area in the future.

Two included studies [4,5] include approximatively $85 \%$ of all cases, which may noticeably influence the results of this meta-analysis. This has already been discussed in the limitation section of the article as follows: There was still substantial heterogeneity among the included cohort studies. Very heterogeneous populations were included in both retrospective and prospective cohort studies.

We agree with Dr. Jourffroy et al. that the infectious agent itself influences the outcomes of septic patients. We attempted to convey the message that culture positivity or negativity was not associated with mortality of sepsis or septic shock patients. A positive culture does not mean a severe infection, and a negative culture does not mean a mild infection. The clinical outcomes may be associated with not only the infection sources but also the management of the sepsis and septic shock.

\section{Acknowledgements}

None.

Authors' contributions

$\mathrm{RJ}$ and $\mathrm{BV}$ wrote and revised the manuscript. Both authors read and approved the final manuscript.

Funding

None.

Availability of data and materials

Not applicable.

\section{Declarations}

Ethical approval and consent to participate Not applicable.

\section{Consent for publication}

$\mathrm{RJ}$ and $\mathrm{BV}$ consent for publication.

Competing interests

$\mathrm{RJ}$ and $\mathrm{BV}$ have no conflicts of interest to declare.

\section{Author details}

${ }^{1}$ Service de médecine intensive réanimation, Hôpital Universitaire Ambroise Paré, Assistance Publique - Hôpitaux de Paris, and Paris Saclay University, Paris, France. ${ }^{2}$ SAMU de Paris, Service d'Anesthésie Réanimation, Hôpital Universitaire Necker - Enfants Malades, Assistance Publique - Hôpitaux de Paris, and Paris University, Paris, France.

Received: 25 May 2021 Accepted: 4 June 2021

\section{References}

1. Li Y, Guo J, Yang H, Li H, Shen Y, Zhang D. Comparison of culture-negative and culture-positive sepsis or septic shock: a systematic review and meta-analysis. Crit Care. 2021;25(1):167.

2. Vincent JL, Rello J, Marshall J, Silva E, Anzueto A, Martin CD, Moreno R, Lipman J, Gomersall C, Sakr Y, Reinhart K; EPIC II Group of Investigators. International study of the prevalence and outcomes of infection in intensive care units. JAMA. 2009;302(21):2323-9.

3. Singer M, Deutschman CS, Seymour CW, Shankar-Hari M, Annane D, Bauer M, Bellomo R, Bernard GR, Chiche JD, Coopersmith CM, Hotchkiss RS, Levy MM, Marshall JC, Martin GS, Opal SM, Rubenfeld GD, van der Poll T, Vincent JL, Angus DC. The Third International Consensus Definitions for Sepsis and Septic Shock (Sepsis-3). JAMA. 2016. 23;315(8):801-10.

4. Kethireddy S, Bilgili B, Sees A, Kirchner HL, Ofoma UR, Light RB, Mirzanejad Y, Maki D, Kumar A, Layon AJ, Cooperative Antimicrobial Therapy of Septic Shock (CATSS) Database Research Group, et al. Culture-negative septic shock compared with culture-positive septic shock: a retrospective cohort study. Crit Care Med. 2018;46(4):506-12.

5. Sigakis MJG, Jewell E, Maile MD, Cinti SK, Bateman BT, Engoren M. Culture-negative and culture-positive sepsis: a comparison of characteristics and outcomes. Anesth Analg. 2019;129(5):1300-9.

\section{Publisher's Note}

Springer Nature remains neutral with regard to jurisdictional claims in published maps and institutional affiliations. 